\title{
Information and Power Simultaneously Transmission in Space Shift Keying Modulation System with Antenna Selection
}

\author{
Fuqiang Wang ${ }^{1, a}$, Guangchi Zhang ${ }^{1, b}$ \\ ${ }^{1}$ School of Information Engineering, Guangdong University of Technology, Guangzhou, 510000, \\ China \\ aemail: denghuozhaoyiren@126.com, bemail: gczhang@gdut.edu.cn
}

\begin{abstract}
Keywords: Energy harvesting; Space Shift Keying; antenna selection; exhaustive search; maximum likelihood (ML)
\end{abstract}

\begin{abstract}
In this paper, antenna selection at transmitter in Space Shift Keying (SSK) is considered, which conveying information using the bit-determining antenna from two potential antennas. Previous researches mainly focused on information transmission, and the main performance metrics are bit error rate (BER) and capacity. Simultaneous wireless information and power transmission (SWIPT) has recently drawn significant research attention. In SWIPT systems, receiver can not only receive information but also harvest energy. Wireless energy harvesting (EH) has been proposed to prolong the operation of energy-constrained wireless networks. Combining SSK and $\mathrm{EH}$ together in our work, the main challenge is to find the best antenna combination. For this, we put forward an objective function to get the tradeoff between BER and EH. Exhaustive search is firstly adopted to accomplish the task, and it shows the scheme can get the best result at the cost of high complexity which may not be affordable when the transmit antenna number is large. To lower the complexity, three other criterions are proposed to work the result out rapidly with lower computation complexity while not deteriorating the BER performance much.
\end{abstract}

\section{Introduction}

Multiple-input-multiple-output technology (MIMO) is the candidate for the next generation to meet the sharp increase in data rate, as it transmits multiple data streams on multiple antennas simultaneously without occupying wider bandwidth. So with the help of MIMO technology, we can get larger channel capacity and higher spectral efficiency at the cost of higher complexity at both transmitter and receiver. To improve the performance of reliability and efficiency while decrease the complexity in synchronization, decoding and so on, R. Mesleh and H. Haas put forward the spatial modulation (SM) in [1]. In SM scheme for MIMO system, the information is conveyed in two ways, i.e., the constellation of amplitude/phase modulation (APM) and the signal constellation formed by channel response. Spatial modulation has been shown to be very promising as a low complexity MIMO communication solution for its capability to exploit MIMO capacity utilizing only one RF chain at the transmitter. The performance of spatial modulation has been extensively studied for different fading channels [2]. One of the simplest SM is Space shift keying (SSK) in which one single transmit antenna is activated in each slot to transmit a given signal according to the bit streams. That is to say, all the bits are conveyed by the index of antenna.

Simultaneous wireless information and power transmission (SWIPT) has recently drawn significant research attention. In SWIPT systems, receiver can not only receive information but also harvest energy [3] [4]. Wireless energy harvesting (EH) has been proposed to prolong the operation of energy-constrained wireless networks [5] [6] [7].The power management problem for the point -to-point communication powered by EH sources is studied in [4]-[6] with the deterministic EH model, and in [7] [9] with the random EH model. The authors in [6]-[10] studied the throughput maximization problems for the additive white Gaussian noise (AWGN) channel. Considering the finite energy storage limit, the results in [6] were generalized in [8]. The AWGN channel capacity under EH constraints is studied in [10], and it turns out that the same capacity can be achieved with the time-varying energy source. EH receiver and information receiver can be co-located or 
distributed and the later is adopted in system model.

As SSK activates one single transmit antenna in each time slot to convey information [13-14], and the signal constellation is only formed by the channel response of each transmit antenna, we can choose two possible links among antennas more than two to get the proper tradeoff between EH and BER. That's to say, we can get different harvested energy under the same BER, and vice versa. Euclidean distance between two channel gains has an effect on the performance, which is shown in [12] that the better BER can achieved with larger Euclidean distance between two channel gains. With the intention of transmitting information while considering power simultaneously, we design an objective function to form the tradeoff between BER and EH by exploiting proper antenna combination. To find the desired antenna combination, exhaustive search is adopted first. However, the scheme can get the best result at the cost of high complexity which may be affordable for receiver sometimes. To lower the complexity, three other criterions are proposed to lower the computation complexity while not deteriorating the BER performance much. In scheme 1, a tradeoff is firstly made between the gain of information channel and power channel to get one potential antenna and the other with the objective function. The information channel gain and power channel gain are only first considered respectively, and then the final antenna combinations are obtained with the help of the objective function in scheme 2 and scheme 3. Compared with the exhaustive search to get the antenna index, the other three schemes are less complex in calculation as the total antenna number increases. The remainder of the paper is organized as follows. In part II, the model of multiple-antenna selection of SSK (MAS-SSK) system is introduced. Four schemes to achieve the tradeoff between energy harvesting and bits error rate are provided in part III. Simulation results and performance analysis are given in part IV. Conclusion appears in part V.

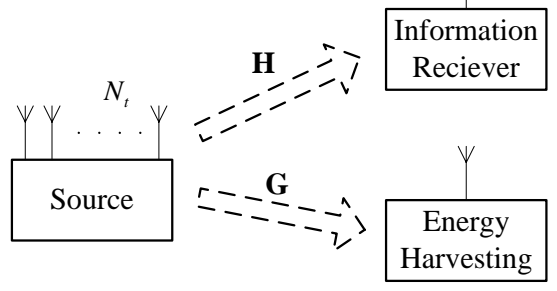

Fig.1 SSK information transmitting with EH

\section{System Model of MAS-SSK}

We consider a multiple-input-single-output (MISO) system with $N_{t}$ transmitting antennas at transmitter and $N_{r} \quad\left(N_{r}=1\right)$ receiving antennas at both information receiver and energy harvesting receiver, as shown in Fig.1. In contrast to conventional SSK which uses the same potential antenna combination to convey information, in the proposed MAS-SSK scheme, the potential antenna combination is changeable according to different schemes. The information receiver and power receiver are distributed in the model system; we can get channel state information (CSI) at receiver through channel estimation and sends the optimal antenna combination to transmitter through a low-bandwidth feedback path $[15,16]$. According to the coming data, the transmitter activates one antenna from the antenna combination of two potential antennas. The data streams are transmitted through a flat fading channel $h_{k} \in \mathbf{H}^{N_{t} \times 1}$ from source to information receiver by the $\mathrm{k}$-th active antenna and $g_{k} \in \mathbf{G}^{N_{t} \times 1}$ stands for the channel from source to energy receiver with the same antenna, where both $\mathbf{H}$ and $\mathbf{G}$ are a vector with $N_{t}$ elements. The the received signal at the information receiver can be formed as

$$
y=\sqrt{\rho} h_{k} x+n_{k}
$$

The signal at the energy receiver is

$$
y_{E H}=\sqrt{\rho} g_{k} x+n_{e k}
$$


and the harvested energy power is

$$
p_{E H}=P_{0}\left\|g_{k}\right\|^{2}
$$

where $x \in\{0,1\}, n_{k}$ and $n_{e k}$ is the additive white Gaussian noise (AWGN) which are independent and identically distributed (i.i.d.) according to $C N(0,1)$. Besides, the channel gains $h_{k}$ and $g_{k}$ follow complex Gaussian distribution with zero mean and variance $N_{0} \cdot \rho$ is the average signal to noise ratio (SNR), and $\rho=\frac{P_{0}}{N_{0}}$, where $P_{0}$ is the transmitting power and $N_{0}$ is the power of noise. Assumption of perfect CSI at transmitter and receiver is provided in the paper. The maximum likelihood (ML) criterion is adopted for the information detection (ID) at information receiver whose formula can be (4).

$$
\tilde{i}=\underset{i . i=1, \ldots, N t}{\arg \min }\left\|y-h_{i} s\right\|
$$

where $\tilde{i}$ means the active antenna index decoded in ML method at information receiver.

\section{Tradeoff between BER and EH under Different Schemes}

In this section, we first introduce the motivation for the work and propose the exact objective function. Trying to find the solution to the function, exhaustive search is discussed firstly. In order to avoid the prohibitive complexity of exhaustive search, we proposed three greedy antenna selection algorithm for the optimization problem defined in Eq.(7). Finally, we present the computational complexity analysis of different algorithms.

Previous researches on SSK mainly focused on the performance of BER or energy harvesting (EH) which is so important in improving the energy efficiency, but no one taking them into consideration at the same time. In [12], (5) is proven that the better BER can be achieved with larger Euclidean distance between two channel gains which is expressed as $\left\|h_{i}-h_{j}\right\|^{2}$ as follows.

$$
P_{b} \leq \frac{1}{2\left(N_{t}-1\right)} \sum_{i=1}^{N_{t}} \sum_{j=1, j \neq i}^{N_{t}} Q\left(\frac{\sqrt{P_{0}}\left\|h_{i}^{N_{t}}-h_{j}^{N_{t}}\right\|}{2 \sqrt{N_{0} / 2}}\right)
$$

As we can see in equation (5), the desired communication quality, such as BER, can be met with certain Euclidean distance between information channel $h_{j}$ and $h_{i}$. Thus we can take the energy harvesting channel gain into consideration when the least Euclidean distance of information channel is achieved, that's to say, criterion (5) for $\tilde{i}$ is adopted as much as possible.

$$
\tilde{i}=\underset{i . i=1, \ldots, N t}{\arg \max } \rho\left\|g_{k}\right\|^{2}
$$

Motivated by the ideas above and SWIPT, we design an objective function (6) to balance the performance of BER and EH. Above all, a variable $\alpha(\alpha \in[0,1])$ is introduced, so that we can achieve the tradeoff between BER and EH with the help of the objective function (6), where two antenna indexes are produced.

$$
\left[i^{*}, j^{*}\right]=\underset{i, j . i, j=1, \ldots, N t, i \neq j}{\arg \max } \alpha\left\|h_{i}-h_{j}\right\|^{2} * P_{0}+(1-\alpha)\left(\left\|g_{i}\right\|^{2}+\left\|g_{j}\right\|^{2}\right)^{*} P_{0}
$$

For $P_{0}$ is the transmitting power, expression of (6) can be easily rewritten as follows:

$$
\left[i^{*}, j^{*}\right]=\underset{i, j . i, j=1, \ldots, N t, i \neq j .}{\arg \max } \alpha\left\|h_{i}-h_{j}\right\|^{2}+(1-\alpha)\left(\left\|g_{i}\right\|^{2}+\left\|g_{j}\right\|^{2}\right)
$$

Compared with traditional criterion in $[2,13,16]$, choosing antenna indexes according to the maximum Euclidean distance can get better BER performance [12]. $\left\|g_{i}\right\|^{2}+\left\|g_{j}\right\|^{2}$ means the channel gain at energy receiver. The power of the transmitted signal $s$ is $P_{0}$.As the variable $\alpha$ changes, the weighting efficient changes. When $\alpha$ grows, more attention is paid to energy harvesting. Energy harvesting is only considered when $\alpha=0$, information transmission is 
only considered when $\alpha=1$.

Above all, exhaustive search algorithm is used for solving the objective function to find the optimal antenna combination. As exhaustive search algorithm shows below, the antenna combination maximizing the objective function is produced though the search for all the possible combinations. Undoubtedly, we can get the optimal result, while enduring the high complexity of the algorithm whose plural multiplication is $N_{t}\left(N_{t}+1\right) / 2$.

In order to avoid searching all the antenna combinations with high complexity, three low-complexity greedy antenna selection schemes are given below. We can work the result out rapidly with lower computation complexity in the three proposed algorithms while not deteriorating the BER performance much. The complexity of the schemes is compared at the end of the part.

In scheme 1, one of the desired antenna indexs is formed according to the following formula Eq.(9).

$$
i^{*}=\underset{i}{\arg \max } \alpha\left\|h_{i}\right\|^{2}+(1-\alpha)\left\|g_{i}\right\|^{2}
$$

which means maximizing the sum of channel gains between information and power transmission, and the other one antenna index is achieved though the objective function (9) with one antenna fixed .

$$
j^{*}=\underset{j . j \neq i^{*}, j=1, \ldots, N t}{\arg \max } \alpha\left\|h_{i^{*}}-h_{j}\right\|^{2}+(1-\alpha)\left(\left\|g_{i^{*}}\right\|^{2}+\left\|g_{j}\right\|^{2}\right) .
$$

The maximal information channel gain is help of the formula (10) in Scheme 2 to

employed to get the first antenna with the

$$
i^{*}=\underset{i . i=1, \ldots, N t}{\arg \max }\left\|h_{i}\right\|^{2}
$$
enhance the information channel gain,

and the other one with the objective function (9) when $i *$ is fixed. Both the scheme 1 and scheme 2 are of the same plural multiplication $3 N_{t}-1$.

Unlike scheme 2, we take the channel gain related to energy transmission as the criterion to select the first antenna index in scheme 3. By employing the formula

$$
i^{*}=\underset{i . i=1, \ldots, N t}{\arg \max }\left\|g_{i}\right\|^{2}
$$

the first antenna index is gained, and the other though the objective function (9). As simulation results show that the scheme has the least times in plural multiplication $2 N_{t}-1$.

The four schemes are provided above, and their complexity in antenna selection is considered respectively. Take the number of plural multiplication for example. As shown in table 1, it needs the least time to get the antenna index in scheme 3 all the time for a given $\alpha$. Exhaustive search is less complex than scheme 1 and scheme 2 when the total number of transmitting antenna is not more than 4.However, the geometric growth in plural multiplication calculation brings heavy burden to the receiver in choosing the best antenna index when the number of transmitting antenna is no less than 5 . In relative terms, scheme 1 and scheme 2 have the same scale in calculation which is far less than the exhaustive search when $N_{t}$ is large.

Table 1 .Complexity Comparison in Plural Multiplication for Different Algorithms

\begin{tabular}{lcccc}
\hline & $\begin{array}{c}\text { Exhaustive } \\
\text { Search }\end{array}$ & Scheme 1 & Scheme 2 & Scheme 3 \\
\hline $\begin{array}{l}\text { Times of plural } \\
\text { multiplication }\end{array}$ & $N_{t}\left(N_{t}+1\right) / 2$ & $3 N_{t}-1$ & $3 N_{t}-1$ & $2 N_{t}-1$ \\
\hline
\end{tabular}

\section{Simulation Results and Performance Analysis}

In this section, we provide simulation results to demonstrate the potential of the proposed algorithms. Performance of EH and BER for different schemes is gained over $10^{6}$ channel realization. $\rho=\frac{P_{0}}{N_{0}}=15 \mathrm{~dB}$ is assumed (power of noise $N_{0}=1 \mathrm{dBm}$ ) to get the tradeoff performance 
in Fig6-7. The number of antennas for transmitting information is set to be $N t=4,8$, and the number of antennas for information receiver is $N r=1$.

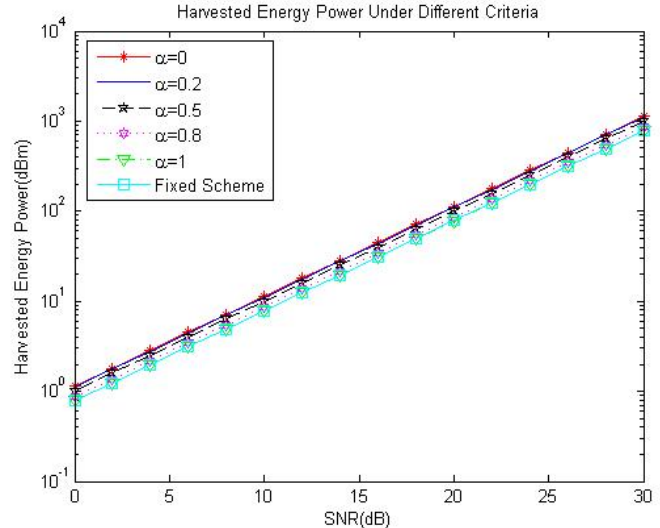

Fig.2 Harvested energy in exhaustive search with various $\alpha\left(N_{t}=4\right)$

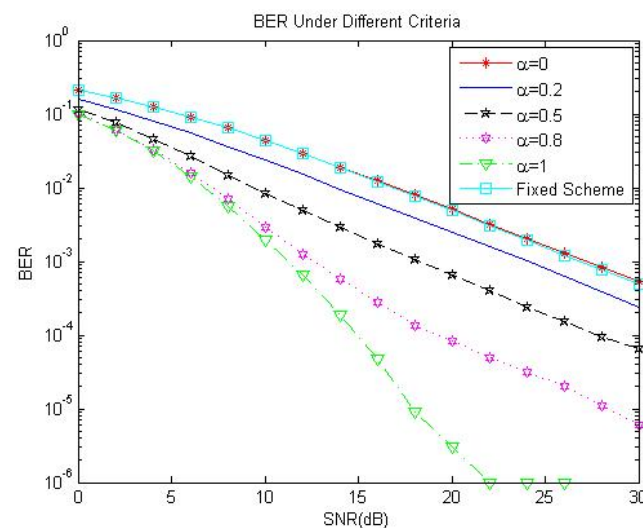

Fig.3 BER in exhaustive search with various $\alpha\left(N_{t}=4\right)$

As figure 2 shows that when $\alpha$ equals to zero, what we consider is just about the energy channel gain where $N t=4$. Thus we can get the largest energy gain while the responding worst BER which is shown in figure 3. The performance of BER and EH when $\alpha=0$ is the same with traditional SSK where no changeable antenna combination is adopted. As the weighting factor $\alpha$ increases, the harvested energy becomes less while the performance of BER gets better. To observe the effect of transmitting antenna number, simulation results with $N t=8$ is provided as follows.

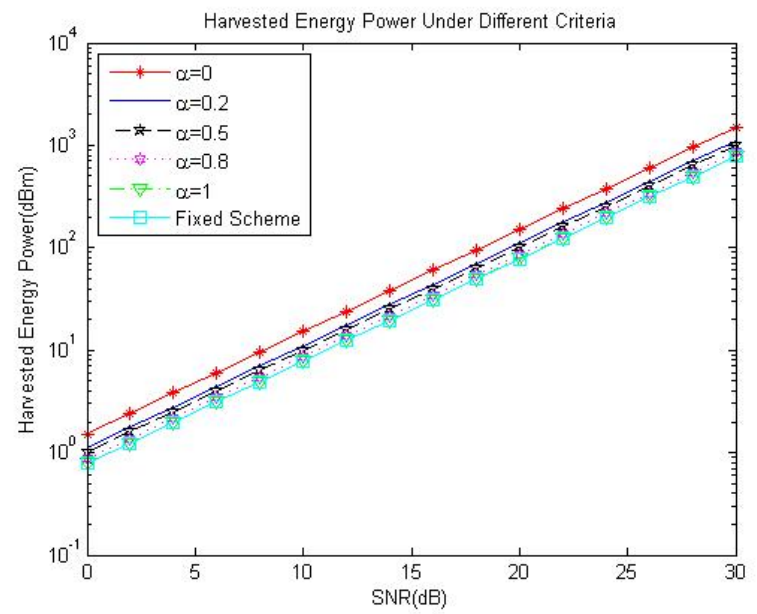

Fig.4 Harvested energy in exhaustive search with various $\alpha$ when $N t=8$

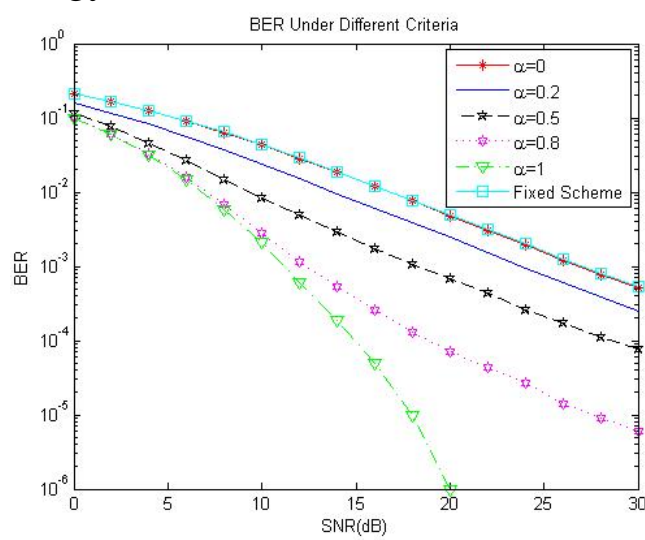

Fig.5 BER in exhaustive search with various $\alpha$ when $N t=8$ 
Compared to the results above, it's indicated in Fig. 5 that except the cases $\alpha=0$ and the fixed scheme, the performance of BER becomes better for other cases, which is obviously shown when $\alpha=1$ where no error bits are detected as soon as SNR reaches $20 \mathrm{~dB}$. As no attention is paid to the information channel in the cases $\alpha=0$ and the fixed scheme, the performance of BER keeps invariant. However, there is about $3 \mathrm{~dB}$ increase in harvesting energy in case $\alpha=0$. Both of the performance EH and BER keeps invariant as no other antenna combination is accessible.

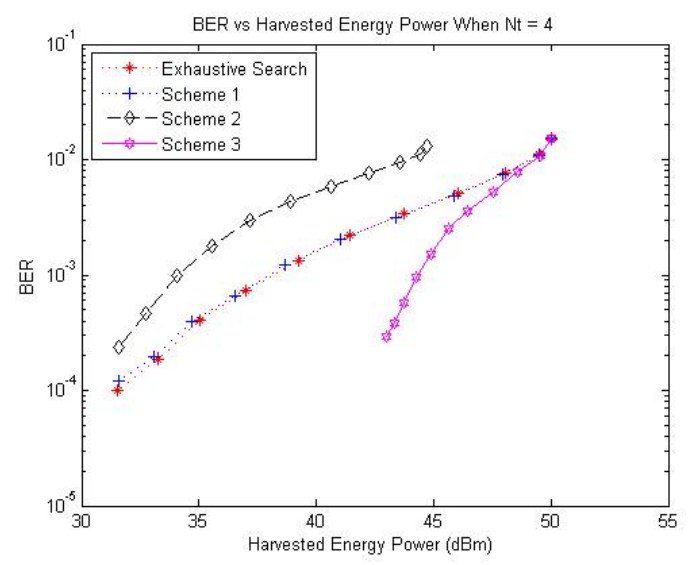

Fig.6 EH vs. BER with various $\alpha(N t=4)$

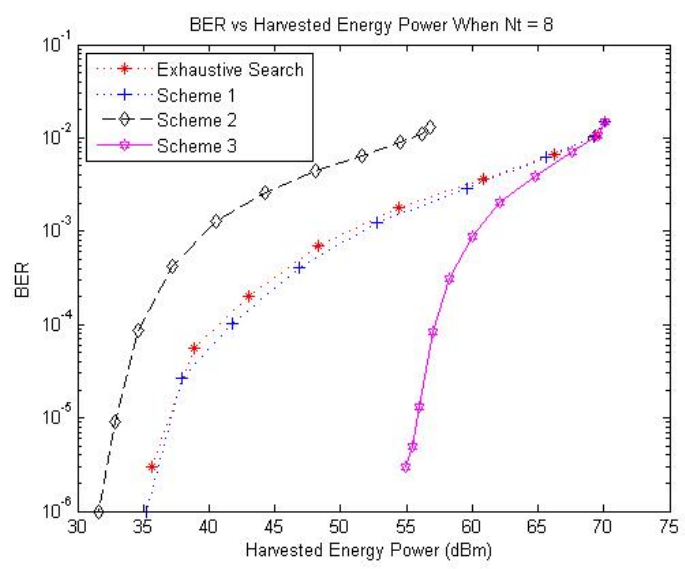

Fig.7 EH vs. BER with various $\alpha(N t=8)$

In Fig.6, we draw the performance of BER and EH simultaneously when the transmitted power is 15 and there are 4 antennas at transmitter. As depicted in Fig.6 scheme 1 and exhaustive search have similar performance which is better than that in scheme 2. We can get Fig.7 with different transmitting antennas in the same way. What we can see in figure 6-7 is that as $N t$ increases, both the performance of $\mathrm{EH}$ and BER get better obviously at the same time for more antenna combinations with larger channel gain are available. In each of the figures from figure 6 to figure 7, the performance of BER gets worse as $\alpha$ decreases. $\mathrm{EH}$ in exhaustive search and scheme 1 to scheme 2 has the same performance for different schemes even when $N t$ varies while $\alpha=1$ for no attention is focused on EH. Therefore, there must be a balance point between EH and BER to meet the required communication quality while getting more energy.

\section{Conclusion}

Four different transmit selection schemes for SSK modulation are provided in this paper. The schemes except the exhaustive search scheme we proposed have lower computation complexity and better performance than that of traditional schemes. The tradeoff between harvested energy and bits error rate is given to show the contradictory relationship. We can change the $\alpha$ to meet the required communication parameters while harvesting more energy.

\section{Acknowledgments}

This work was supported by the National Natural Science Foundation of China under Grand 61571138, the Natural Science Foundation of Guangdong Province under Grand 2015A030313481, the Scientific and Technological Project of Guangzhou City under Grand 2013J2200071, the Scientific Innovation Project of the Education Department of Guangdong Province under Grand 2013KJCX0060, and the Scientific Talent Development Project of Guangdong University of Technology under Grand 220411321.

\section{References}

[1] R. Mesleh, H. Haas, S. Sinanovic, C. W. Ahn, and S. Yun, “Spatial 'Modulation,” IEEE Trans. 
Veh. Technol., vol. 57, no. 4, pp. 2228 -2241, July 2008.

[2] Di Renzo, Marco; Haas, Harald; Ghrayeb, Ali; Sugiura, Shinya; Hanzo, Lajos, "Spatial Modulation for Generalized MIMO: Challenges, Opportunities, and Implementation” ,Proceedings of the IEEE,10.1109/JPROC.2013.2287851,pp.56-103 Jan. 2014 .

[3] L. R. Varshney, “Transporting information and energy simultaneously,”in Proc. 2008 IEEE Int. Symp. Inf. Theory, pp. 1612-1616.

[4]S. Sudevalayam and P. Kulkarni, "Energy harvesting sensor nodes: survey and implications," IEEE Commun. Surveys Tuts., vol. 13, no. 3, pp. 443-461, 2011.

[5] Fengchao Zhul, Feifei Gao, and Minh Yao, "A New Cognitive Radio Strategy for SWIPT System,” 2014 International Workshop on High Mobility Wireless Communications (HMWC'2014).

[6]J. Yang and S. Ulukus, "Optimal packet scheduling in an energy harvesting communication system,” IEEE Trans. Commun., vol. 60, no.1, pp. 220-230, Jan. 2012.

[7]C. K. Ho and R. Zhang, "Optimal energy allocation for wireless communications with energy harvesting constraints,” to be published.[Online]. Available: arXiv:1103.5290

[8]K. Tutuncuoglu and A. Yener, "Optimum transmission policies for battery limited energy harvesting nodes,” IEEE Trans. Wireless Commun., vol. 11, no. 3, pp. 1180-1189, Mar. 2012.

[9]O. Ozel and S. Ulukus, "Information-theoretic analysis of an energy harvesting communication system,” in Proc. IEEE Personal, Indoor Mobile Radio Conf. (PIMRC), Sep. 2010.

[10]Chuan Huang, Rui Zhang, and Shuguang Cui, "Throughput Maximization for the Gaussian Relay Channel with Energy Harvesting Constraints, "IEEE Journal on Selected Areas in Communications, VOL. 31, NO. 8, AUGUST 2013

[11] L. Liu, R. Zhang, and K. C. Chua, "Wireless information transfer withopportunistic energy harvesting,” IEEE Trans. Wireless Commun., vol. 12,no. 1, pp. 288-300, Jan. 2013.

[12] EnzhiZhou; Li Hao,“Transmit Antenna Selection in Space Shift KeyingModulation, ” Vehicular Technology Conference (VTC Spring), 2013 IEEE 77thpp: 1 - 5,

[13] J. Jeganathan, A. Ghrayeb, L. Szczecinski, and A. Ceron, "Space shift keying modulation for MIMO channels,” Wireless Communications,IEEE Transactions on, vol. 8, no. 7, pp. 3692 -3703, 2009.

[14] M. Di Renzo and H. Haas, "Bit Error Probability of Space-Shift Keying MIMO Over Multiple-Access Independent Fading Channels,” Vehicular Technology, IEEE Transactions on, vol. 60, no. 8, pp. 3694-3711, 2011.

[15]M. M. U. Faiz, S. Al-Ghadhban, and A. Zerguine, "Recursive Least-Squares Adaptive Channel Estimation for Spatial Modulation Systems,”in IEEE Malaysia Int. Conf. Commun., Dec. 2009, pp. $1-4$.

[16]M. Di Renzo and H. Haas, "Space Shift Keying (SSK) Modulation with Partial Channel State Information: Optimal Detector and Performance Analysis over Fading Channels,” IEEE Trans. on Commun., vol. 58,no. 11, pp. 3196 -3210, 2010. 